\title{
Alternativas para reconstrucción auricular con cartílago autólogo.
}

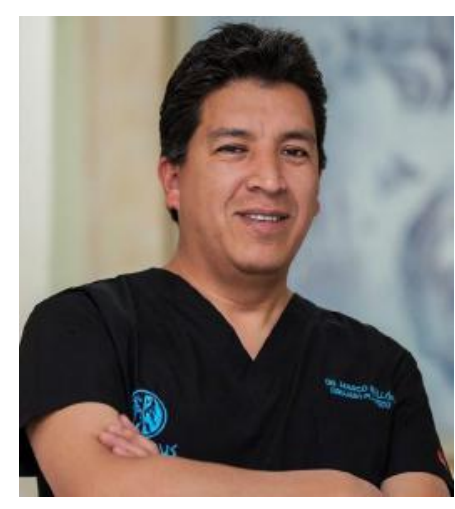

Dr. Marco Ballón

Cirujano Plástico

mballont@yahoo.com

\section{"Auricular reconstruction alternatives, autologous cartilage method"}

Palabra Claver: Reconstrucción, cartílago, autólogo.

Key Words: Reconstruction, cartilage, autologous

\section{RESUMEN}

Debido a la situación anatómica en que se encuentra el pabellón auricular es susceptible a múltiples traumatismos que van desde pequeñas lesiones hasta incluso pérdida total del mismo, dependiendo de la extensión de la lesión podemos decidir tanto la técnica, como el material a utilizar, siendo por estas características un desafío apasionante para el cirujano que puede elegir entre las múltiples opciones que existen para lograr obtener buenos resultados y sin complicaciones.

\section{INTRODUCCIÓN}

La reconstrucción auricular fue descrita por primera vez por Taggliacozzi quien en 1597 describe la plastia con colgajos retroauriculares para región superior e inferior de oreja, más tarde en 1845 Diffenbach dió a conocer la plastia del tercio medio a través de un colgajo de avance, técnica que actualmente sigue siendo aplicada (1), pero fué Burt Brent quien sentó las bases de reconstrucción auricular (2).

Las técnicas de reconstrucción del pabellón auricular han ido evolucionando en el tiempo, la importancia de su conocimiento radica en la frecuencia de afectación, ya sea por un traumatismo, defecto congénito o neoplasia (3). Así mismo constituye un reto para el especialista, debido

\section{ABSTRACT}

Due to the anatomical situation in which the auricle is located, it is susceptible to multiple traumas ranging from small injuries to even total loss, depending on the extent of the injury; we can decide the technique and the material to be used. These characteristics are an exciting challenge for the surgeon who can choose between the multiple options that exist to achieve good results and avoiding complications.

a la complejidad anatómica y necesidad de lograr que el resultado se acerque a lo más normal posible.

La irrigación del pabellón auricular depende principalmente de la arteria Carótida externa, que por medio de dos ramas anastomóticas confiere un sistema de irrigación efectivo. Por otro lado la inervación depende, en región posterior y lóbulo del Nervio Occipital Menor y Nervio Facial, en región anterior y trago por el Nervio Trigémino y por último en región inferior y preauricular por el Nervio Auricular mayor (3).

Las amputaciones post traumáticas generalmente se localizan en el borde libre de la oreja afectando a tres 
planos, dos cutáneos y uno fibrocartilaginoso. El principio de la reconstrucción tras una amputación es reproducir los relieves mediante un soporte cartilaginoso y cubrirlo con un colgajo retroauricular, posteriormente en un segundo tiempo quirúrgico crear el surco retroauricular. Hasta el momento los cartílagos autólogos son el material más confiable para la reconstrucción, el dilema se encuentra en la elección del mismo considerando el fibrocartílago o cartílago costal. (4)

Fibrocartilago: La zona donante ideal es la concha, pues el material es parecido al que se sustituye y es fácil de extraer, las limitaciones se dirigen en torno al tamaño de la misma en comparación con la lesión. $(2,4)$

Cartílago costal: La gran ventaja de su obtención es que la extensión de la zona donante no limita la cantidad de cartílago que puede obtenerse. $(2,4)$

Para efectuar una correcta reconstrucción debemos tomar en cuenta además la localización y extensión del daño. Consideramos para la localización la base, antihélix, hélixy el complejo trago-antitrago y determinamos para la extensión si existe compromiso solamente de piel o también cartílago. Entonces por ejemplo el fibrocartílago de la concha se puede utilizar solo cuando la perdida de sustancia no sobrepasa un cuarto de la oreja y cuando no ocupa más de dos planos adyacentes, de lo contrario se considera el uso del cartílago costal. (2)

Para realizar la reconstrucción auricular debe en primer lugar analizarse todas las lesiones, tratar las prioritarias y analizar la reserva cutánea susceptible de cubrir el injerto cartilaginoso, para lo cual se requieren generalmente dos tiempos quirúrgicos.

Primer tiempo: Consiste en la reconstrucción de los relieves en el cartílago y colocación del mismo recubierto de piel retroauricular.

Segundo tiempo: Constituido por la reconstrucción del surco retro auricular.

Las complicaciones pueden alterar la calidad del resultado, dentro de las cuales se encuentran la infección que provoca reabsorción del cartílago y por tanto debe prevenirse, los hematomas que pueden evitarse con correcta hemostasia y se detectan a los largo de los controles post operatorios y por último la exposición del cartílago que es la más frecuente y se produce como resultado de las dos anteriores complicaciones mencionadas. $(2,4)$

El presente artículo pretende mostrar dos técnicas alternativas para la reconstrucción auricular, además del empleo de cartílago autologo como método eficaz y confiable.
Objetivo: Describir dos diferentes técnicas en el manejo de la reconstrucción parcial auricular usando cartílago autologo.

\section{MATERIAL Y MÉTODO}

Se presentan dos casos ambos de pacientes que sufren trauma parcial auricular, en el primero se utiliza cartílago autologo del mismo lugar de la lesión que fue encontrado 4 horas más tarde del incidente, mientras que en el segundo por la pérdida y extensión del daño se recurre a cartílago costal.

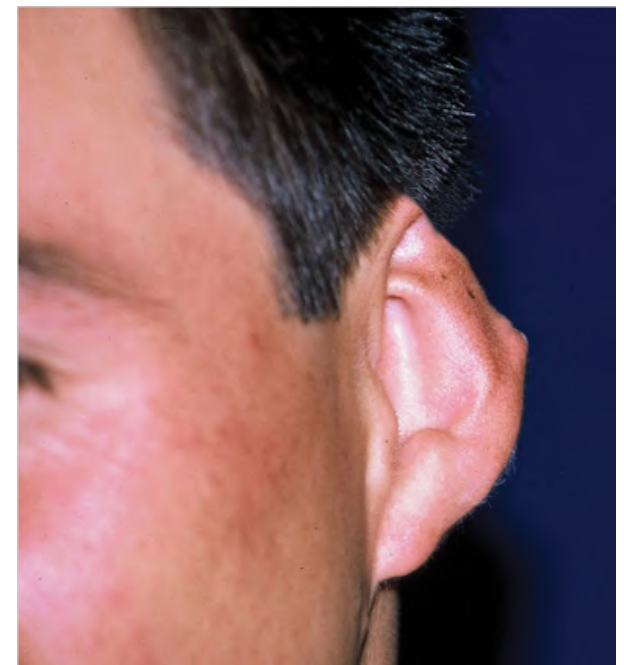

Fig. 1. Vista de pérdida de sustancia en tercio superior de oreja, región de hélix, antihélix y de espesor total segundaria a traumatismo.

\section{PRIMER CASO}
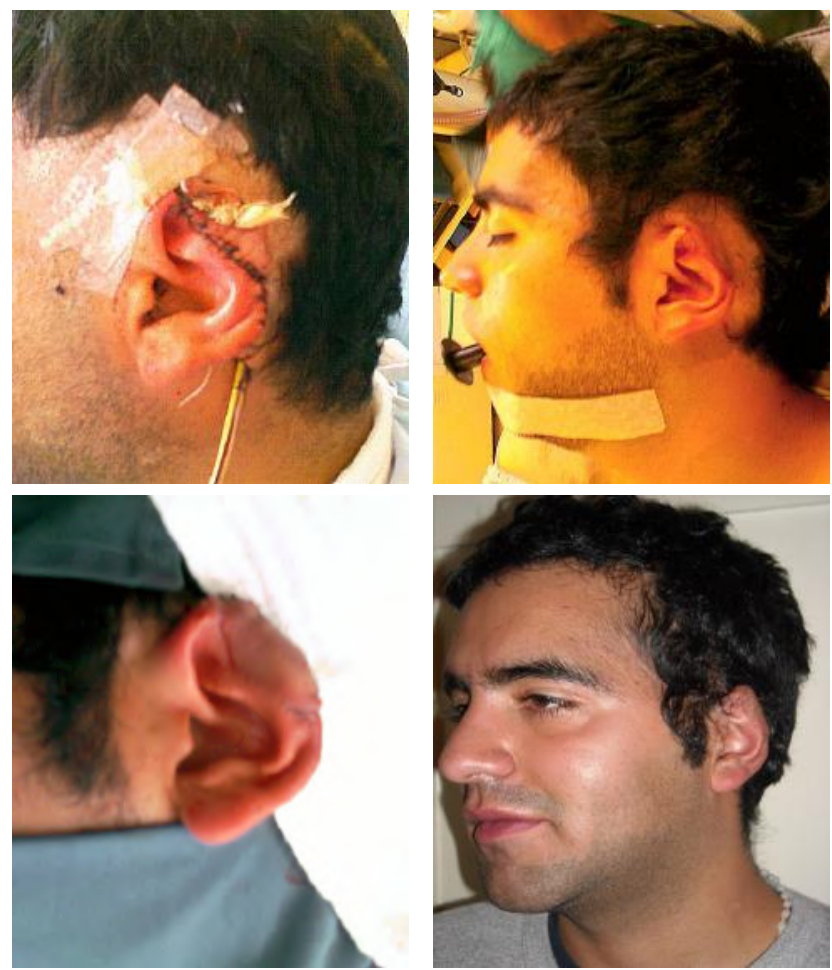

Fig. 2. (A) Colgajo derivado de región mastoidea ipsilateral. (B) Colgajo mastoideo con resultado funcional satisfactorio. (C) Resultado posterior a la sección del pedículo del colgajo. (D) Resultado final. 
Paciente que presenta perdida de sustancia en tercio superior, hélixy antihelix, de espesor total.

Se realiza reconstrucción con cartílago autologo de la misma región seccionada, encontrado 4-5 horas después, en la preparación del mismo se retira piel y pericondrio y se deja en Solución DG-6 por 2 horas, en región de pérdida se regulariza bordes cutáneos y cartilaginosos y se sutura con nylon 5-0 finalmente se cubre con colgajo mastoideo. Luego de 21 días se secciona pedículo del colgajo y se realiza plasta de la misma.

\section{SEGUNDO CASO}

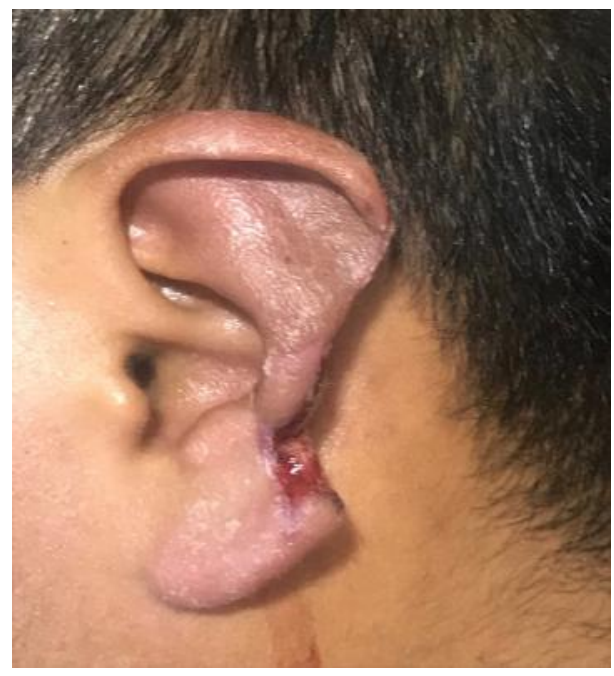

Fig. 3. Pérdida de sustancia en tercio medio de espesor total.
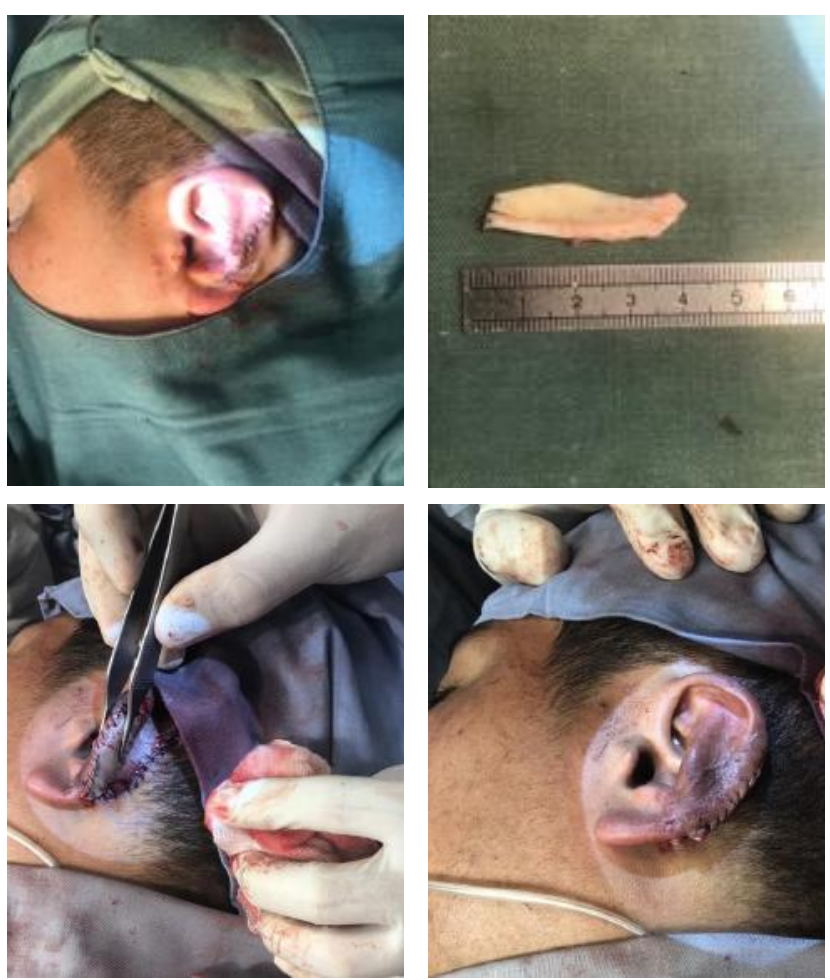

Fig. 4. (A) Pieza de cartilago autologo costal. (B) Colgajo retroauricular. (C) (D) Post operatorio inmediato.
Paciente que presenta perdida de sustancia en tercio medio, hélix y antihelix de espesor total.

Se realiza reconstrucción auricular con cartílago costal, mismo que luego de ser obtenido se talla, formando ausencia de hélix y antihelix, se coloca en zona cruenta uniendo a cartílago y se cubre con colgajo retroauricular, finalmente en vez de realizar plastia en zona cruenta se coloca injerto de piel retroauricular total.

\section{RESULTADOS}

En ambos casos logramos restaurar la anatomía del pabellón auricular, eligiendo en base al grado de afectación y perdida la técnica que nos parecía más adecuada, conservando buena irrigación e inervación y sin presentar complicaciones.

\section{DISCUSIÓN}

Dentro de la reconstrucción auricular existen diversas técnicas que se han desarrollado a lo largo del tiempo, elegiruna muchas veces depende de la habilidad e imaginación del cirujano en base al grado de afectación y materiales con los que cuenta. No obstante, existen técnicas regladas para reconstrucción parcial utilizando cartílago costal para tener un diagnóstico y manejo reconstructivo exacto (2). En el presente artículo se exponen dos casos distintos, el primero usando cartílago autologo amputado de la misma zona y el segundo con cartílago costal, ambos en dos tiempos operatorios respectivamente, pero según la necesidad y magnitud del caso los mismos pueden variar. Se registran casos en los que se necesitan incluso 4 tiempos operatorios dependiendo de la lesión y la magnitud de la misma $(5,6)$.

Hasta el momento los cartílagos autólogos son el material más confiable para la reconstrucción (4) sin embargo métodos más sofisticados utilizan el Polietileno Poroso (Porex) para la reconstrucción parcial o total del pabellón auricular (7). En el estudio en que se compara cartílago con el polietileno poroso, se evidencia que los pacientes que usaron polietileno llegan a tener complicaciones como ser infección y extrusión post operatoria (8), si bien los resultados no muestran grandes diferencias, indican la seguridad del uso de cartílago, que en los dos casos del presente artículo se encontraban disponibles, aunque de distintas fuentes según la lesión.

\section{CONCLUSIÓN}

Por su situación anatómica las lesiones auriculares son relativamente frecuentes en la práctica médica pero todo cirujano plástico verá en algún momento este tipo de lesión, sea traumática, oncológica o congénita y debe estar preparado para resolver el problema de forma aguda, motivo por el cual es importante tener claros los conceptos y opciones a considerar. 
Existen diversas técnicas como se expuso las que se basan en restaurar pliegues y depresiones de oreja, poner buen soporte cartilaginoso con cobertura de piel lo más parecido a la zona amputada respetando siempre las unidades anatómicas. La elección de la técnica dependerá de la extensión de la lesión, los materiales con los que se cuenta y la habilidad del cirujano, teniendo en cuenta el respaldo que se tiene hasta ahora sobre la seguridad y eficacia a momento de usar cartílago autologo sobre otros materiales.

\section{BIBLIOGRAFÍA}

1. Mc. Carthy J.G.: "Cirugía Plástica”. Vol. II. Buenos Aires. Editorial Médica Panamericana. 1992. Pp.1198 - 1213

2. López-Cedrún, J. Cirugía reconstructiva y estética del tercio medio facial. Madrid: Arán Ediciones, Pp.219-246.

3. Schulz M, Salem C. Reconstrucción auricular. Cuadernos de Cirugía 2003;17:Pp. 75-85

4. F. Firmin, A. Marchac. Reconstrucción auricular tras amputaciones traumáticas. Amputaciones parciales y totales o subtotales. EMC - Cirugía Plástica Reparadora y Estética, Volume 21, Issue 2, 2013, 1-21

5. Mantilla, M. Reconstrucción auricular: experiencia de 24 años. Cir. plást. Iberolatinoam, 2008; 34 (2): 34-39.

6. Gantz V José Tomás, de la Cerda A Teresa y cols. Reconstrucción en un tiempo del pabellón auricular en amputación parcial traumática con preservación del pedículo inferior: Revisión de la literatura y presentación de doscasos.Rev.Otorrinolaringol.Cir.CabezaCuello [Internet]. 2015 Dic [citado 2018 Nov 10]; 75(3):265-269. Disponible en: $\quad$ https://scielo.conicyt.cl/scielo.php?script=sci_ arttext\&pid=S0718-48162015000300012\&lng=es. http:// dx.doi.org/10.4067/S0718-48162015000300012.

7. Oztürk S, Sengezer M, Zor F. Reconstruction of acquired partial auricular defects by porous polyethylene implant and superficial temporoparietal fascia flap in adult patients. Plast Reconstr Surg. 2006;118 (6):1349-57.

8. Constantine KK, Gilmore J, Lee K, Leach J. Comparison of Microtia Reconstruction Outcomes Using Rib Cartilage vs Porous Polyethylene Implant. JAMA Facial Plast Surg. 2014;16(4):240-244. doi:10.1001/jamafacial.2014.30 